\title{
Roll Orientation Estimator for Smart Projectiles Using Thermopile Sensors
}

\author{
Jonathan Rogers* and Mark Costello \\ Georgia Institute of Technology, Atlanta, Georgia 30332 \\ and \\ David Hepner‡ \\ U.S. Army Research Laboratory, Aberdeen Proving Ground, Maryland 21005
}

\section{DOI: $\underline{10.2514 / 1.52935}$}

\begin{abstract}
The use of inexpensive, commercially available thermopiles sensors for roll orientation estimation of spinning bodies is explored. The sensors convert observed thermal gradients into an electrical signal well suited for onboard data acquisition and real-time signal processing. An environmental model emulating sensor stimulus for a sixdegree-of-freedom body is generated given standard atmospheric and typical ground conditions. When sensor characteristics are included, the fully developed model can be used to generate accurate sensor output as a function of Euler angles and altitude. Outputs from the model are then shown to compare favorably with experimental flight data, capturing the predominant and nearly sinusoidal signal variation as the projectile rolls. An extended Kalman filter algorithm is offered, which enables real-time roll angle and roll rate estimation using solely thermopiles as feedback. Example results demonstrate that the algorithm yields reasonably accurate roll information. Finally, a trade study demonstrates that roll error is further mitigated as the number of thermopile sensors is increased. This research shows that thermopiles could be useful in a diverse multisensor constellation as a convenient absolute inertial roll reference.
\end{abstract}

\section{Nomenclature}

$\mathbf{I}_{I}, \mathbf{J}_{I}, \mathbf{K}_{I}=$ inertial reference frame unit vectors

$\mathbf{I}_{P}, \mathbf{J}_{P}, \mathbf{K}_{P}=$ projectile reference frame unit vectors

$k_{v} \quad=$ sensor scale factor

$P \quad=$ roll angle error covariance estimate

$Q \quad=$ model error covariance for Kalman filter

$R=$ measurement error covariance for Kalman filter

$T=$ infrared transmission coefficient

$V=$ thermopile output signal

$x_{e}, y_{e}, z_{e}=$ position coordinates with respect to the inertial frame of the projectile mass center

$y=$ infrared emissivity

$\beta \quad=$ azimuth angle with respect to inertial frame

$\gamma \quad=$ angle with respect to horizon

$\delta_{i} \quad=$ thermopile field-of-view roll angle offset from $\mathbf{J}_{\boldsymbol{P}}$ $\phi, \theta=$ projectile Euler roll and pitch yaw angles

\section{Introduction}

$\mathbf{R}$ EDUCED size combined with increased availability and affordability of commercial sensing and processing technology has enabled munitions designers to consider a wide variety of component-level options toward reduced-state and full-state guidance, navigation, and control (GNC). However, in stark contrast to missiles, gun-launched munitions must withstand extreme loads while in bore and often spin rapidly upon muzzle exit. These dynamic challenges hinder traditional six-degree-of-freedom state estimation that embraces a classic constellation of three accelerometers and

Received 25 October 2010; revision received 20 December 2010; accepted for publication 21 December 2010. This material is declared a work of the U.S. Government and is not subject to copyright protection in the United States. Copies of this paper may be made for personal or internal use, on condition that the copier pay the $\$ 10.00$ per-copy fee to the Copyright Clearance Center, Inc., 222 Rosewood Drive, Danvers, MA 01923; include the code 0731-5090/11 and \$10.00 in correspondence with the CCC.

${ }^{*}$ Research Engineer, School of Aerospace Engineering. Member AIAA.

${ }^{\dagger}$ Sikorsky Associate Professor, School of Aerospace Engineering. Associate Fellow AIAA.

${ }^{\ddagger}$ Senior Research Engineer, Weapons and Materials Research Directorate. three rate sensors. Orientation estimators, in particular, have not translated well from aircraft and missiles since they are too expensive, lack launch robustness, do not fit within the allotted space, or are too application specific. As a result, the smart projectile community is considering component-level integration of low-cost sensors such as accelerometers and rate sensors, magnetometers, and other small robust individual sensor technology as an alternative orientation solution. A component-level integration approach offers significant opportunity for constellation variants across a wide range of platforms, missions, and conditions. In some cases, sensors can perform redundant measurements to increase robustness. In others, sensors can cross-calibrate each other. It is also quite possible that a constellation of sensors provides full coverage while individual devices may be partially compromised by environmental conditions. One such sensor being considered for inclusion in these constellations is thermopiles, which can be used to detect infrared (IR) emissivity gradients between the earth and sky. Whenever conditions are sufficient, a thermopile signal can be processed to generate real-time angle and angular rate estimates, certainly for roll, and possibly for pitch as well.

The use of thermal sensors or horizon sensors to detect differences in IR emissivity between ground and sky is not a new idea, and has been used extensively in the space community for over 50 years. Horizon sensor development began as part of the Jupiter-C rocket reentry experiment, and subsequent horizon sensors were used in the Mercury, Gemini, and other programs. Various sensor designs in several NASA programs demonstrated success in determining vehicle orientation to accuracies of less than half a degree [1]. Since these initial successes, several authors have proposed various methods for incorporating horizon sensors in satellite attitude estimation. Astheimer [2] applied Earth horizon sensing techniques to lunar horizon sensing as well, and characterized errors limiting estimation accuracy. Experimental results from X-15 rocket flights verified that estimation accuracies within $0.06 \mathrm{deg}$ were possible at altitudes of $300 \mathrm{~km}$. Doctor [3] proposed an array of horizon sensors that automatically compensates for variations in the Earth's radiance by providing a reference signal to the horizon detection system. Most recently, Grassi [4] developed a spacecraft attitude determination system that combines magnetometer and horizon sensor feedback.

Use of thermal or horizon sensors for attitude feedback onboard atmospheric flight vehicles has been far more limited. Early missile 
designers considered the use of horizon sensor feedback for attitude control $[5,6]$. However, horizon and other thermal sensors often cannot provide feedback with acceptable error levels for highreliability systems such as missiles or manned aircraft. The primary reason for this is sensor response to unpredictable atmospheric stimuli such as large clouds or fog that can obscure the horizon. Thus most applications of thermal sensors to atmospheric vehicle control systems has occurred in the field of unmanned aerial vehicles, which, like projectiles, must maintain low-cost even at the expense of reliability. Gwozdecki [7] has proposed an aircraft orientation estimator that relies on horizon sensors. More recently, Taylor et al. [8], Cornall et al. [9], and Herrmann and Bil [10] have designed and implemented aircraft stabilization systems using only thermopile sensor feedback. Six thermopiles, mounted in opposite directions along each orthogonal axis, provide feedback to a control system that maintains level flight. Experimental flight data have demonstrated reasonable control performance and reliability in clear weather. An interesting extension of previous horizon sensing research was performed by Chahl et al. [11], who discovered that dragonflies' ocelli organs are horizon sensors used primarily for attitude stability.

The contributions of this paper are two-fold. The first is development of a generic, wide field-of-view (FOV) thermopile model that generates simulated thermopile voltage outputs as a function of angle to the horizon and sensor FOV. The second contribution is the development of an extended Kalman filter that estimates roll angle and roll rate strictly from thermopile feedback for smart projectile applications. The estimation algorithm assumes the thermopile signal varies sinusoidally as the projectile rolls, and is robust to unpredictable changes in the Earth's IR radiance. Furthermore, the algorithm can be run in real-time on modest, midrange embedded processors. The paper begins with a derivation of a generic thermopile model. Then, experimental thermopile results are shown for a mortar projectile flight test. This experimental data are compared with data generated from the analytic model, and reasonable correlation is observed. An extended Kalman filter is then presented, and example simulations show that accurate roll angle and roll rate estimates can be obtained. Finally, a trade study shows that estimation accuracy improves exponentially as more thermopiles are incorporated.

\section{Thermopile Model}

\section{A. Model Development}

Thermopiles, originally invented by Joule to increase the output signal of a thermoelectric sensor, function essentially as a set of thermocouples connected together in series [12]. Their main application is remote temperature detection, since sensor output voltages are proportional to the mid- and far-IR energy impingent on the sensor within its FOV. Because the IR emissivity of the Earth's surface is far higher than the sky (the ground looks "warm" compared

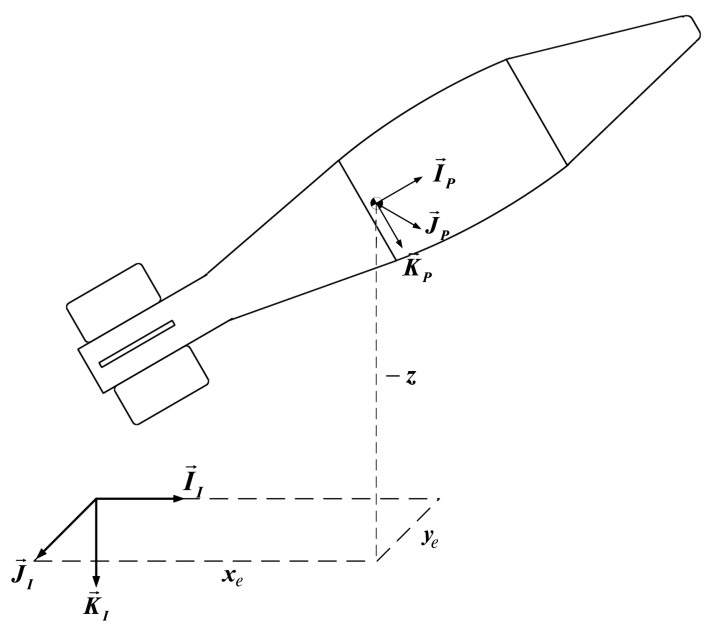

Fig. 1 Reference frame schematic.

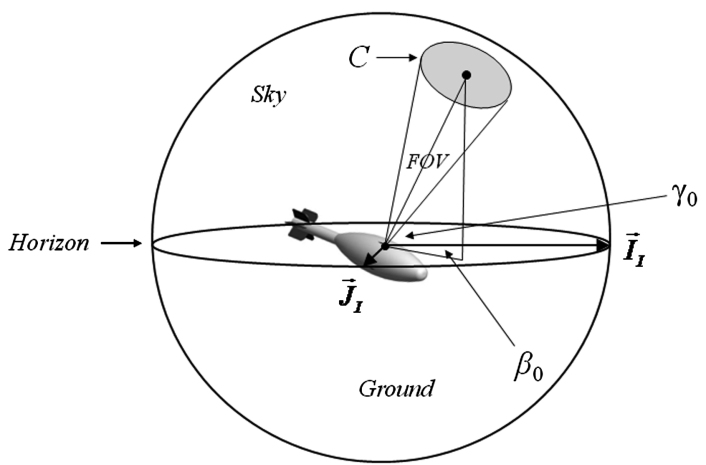

Fig. 2 Projectile-fixed thermopile geometry.

with the sky), thermopile outputs can, at least theoretically, provide an attitude reference with respect to vertical.

To understand sensor response to guided projectile flight environments, it is first helpful to develop a fundamental model of thermopile outputs as a function of sensor orientation with respect to the atmosphere and sensor FOV. Two reference frames will be used throughout this paper: an inertial frame $I$ and a standard body-fixed frame $P$. The body-fixed reference frame is defined according to the standard aerospace Euler angle rotation sequence. Figure $\underline{1}$ shows a diagram of the two relevant reference frames.

Consider the sensor geometry as shown in Fig. 2. A thermopile sensor with a given FOV is fixed on a projectile at the center of a unit sphere. The equator of the sphere represents the Earth's horizon, and the projectile is free to rotate in any direction with respect to the sphere. The FOV bisector has a total inclination angle (zenith angle) with respect to the horizon plane of $\gamma_{0}$ (where $0 \leq \gamma_{0}<2 \pi$ ). The cone representing the thermopile's viewing volume intersects the unit sphere forming a circle $C$. Furthermore, the FOV bisector has an azimuth angle with respect to $\mathbf{I}_{I}$ of $\beta_{0}$.

Thermopile voltage outputs are proportional to the total mid- and far-IR energy impingent on the sensor substrate. Cornall et al. [9] as well as many other authors have shown that the IR radiance of the Earth's atmosphere is at its minimum at zenith angles pointed straight up, and grows exponentially as the zenith angle goes to zero. When the sensor is pointed toward the ground, slant range attenuation effects tend to lower total radiance at angles toward the horizon, and thus radiance is at its peak when the sensor looks straight down. Define a function $y(\gamma)$ on the unit sphere representing the total IR emissivity at each zenith angle given by

$$
y(\gamma)= \begin{cases}k_{1} e^{-\alpha \gamma}+k_{2} & \text { if } 0<\gamma \leq \pi / 2 \\ k_{1} e^{-\alpha(\pi / 2-\gamma)}+k_{2} & \text { if } \pi / 2<\gamma<\pi \\ p_{2} \gamma^{2}+p_{1} \gamma+p_{0} & \text { if } \pi \leq \gamma \leq 2 \pi\end{cases}
$$

where $k_{1}=59.0, k_{2}=6.0, \alpha=-11.0, p_{0}=-55.0, p_{1}=57.3$, and $p_{2}=-6.1$. The exponential coefficients $k_{1}, k_{2}$, and $\alpha$ represent curve fits obtained from [13], while the polynomial coefficients $p_{i}$ were used to generate reasonable slant range attenuation profiles. Note that IR emissivity in general does not change with respect to azimuth angle, and thus $y$ is not a function of $\beta$. Figure $\underline{3}$ shows a plot of $y$ as a function of horizon angle.

Thermopile sensor output voltage is proportional to the IR radiance contained within the contour $C$, and thus can be expressed as

$$
V(\gamma)=k_{v} \iint_{C} y(\gamma) \mathrm{d} \beta \mathrm{d} \gamma
$$

Since $C$ is a circle, Eq. (2) becomes

$$
V(\gamma)=k_{v} \int_{\gamma_{0}-\mathrm{FOV} / 2}^{\gamma_{0}+\mathrm{FOV} / 2} 2 y(\gamma) \sqrt{\left(\frac{\mathrm{FOV}}{2}\right)^{2}-\left(\gamma-\gamma_{0}\right)^{2}} \mathrm{~d} \gamma
$$

Closed-form solutions for Eq. (3) could not be found for the piecewise regions of $y$ defined by exponentials, and thus $y(\gamma)$ is 


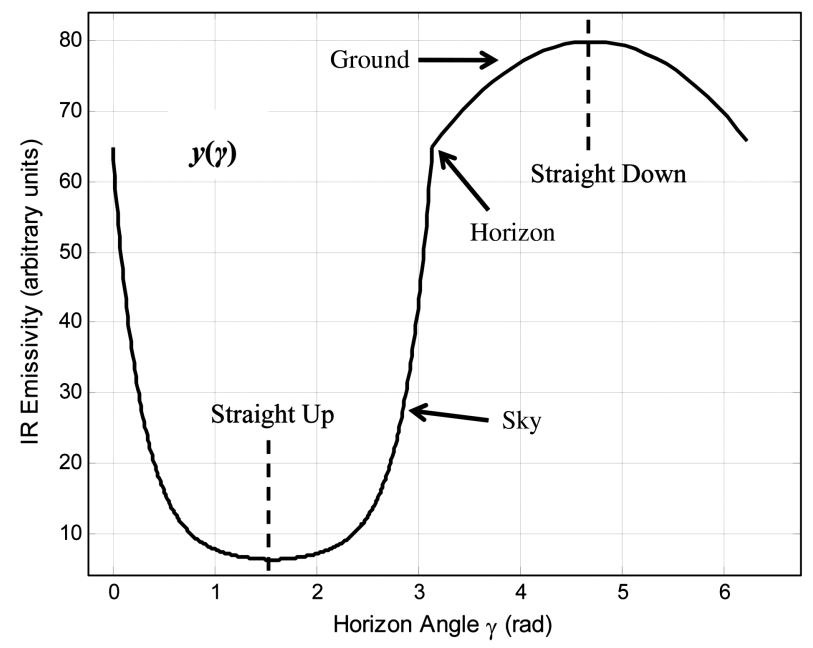

Fig. $3 y(\gamma)$ representing IR emissivity as a function of horizon angle.

redefined such that all exponentials are replaced by fifth-order polynomials, according to

$$
\begin{aligned}
& y(\gamma) \\
& \quad= \begin{cases}q_{5} \gamma^{5}+q_{4} \gamma^{4}+q_{3} \gamma^{3}+q_{2} \gamma^{2}+q_{1} \gamma+q_{0} & \text { if } 0<\gamma \leq \pi / 2 \\
c_{5} \gamma^{5}+c_{4} \gamma^{4}+c_{3} \gamma^{3}+c_{2} \gamma^{2}+c_{1} \gamma+c_{0} & \text { if } \pi / 2<\gamma<\pi \\
p_{2} \gamma^{2}+p_{1} \gamma+p_{0} & \text { if } \pi \leq \gamma \leq 2 \pi\end{cases}
\end{aligned}
$$

All coefficients $q_{i}$ and $c_{i}$ in Eq. (4) are defined in Table 1 . Note that fifth-order polynomials fit the data given in [13] with accuracy comparable to the exponential fits. The expression in Eq. (4) can be used to solve the integral in Eq. (3) for a closed-form solution of thermopile voltage outputs as a function of horizon angle $\gamma_{0}$. This is accomplished by solving the integral in nine different piecewise regions given certain conditions on the horizon angle $\gamma_{0}$ and sensor FOV. By defining these nine regions, the integral in Eq. (3) can be solved in each region. Table 2 describes the conditions defining each region, while expressions for each integral are shown in the Appendix. Figure 4 shows diagrams of sensor orientation for each region. Note that the final closed-form expressions for $V_{i}\left(\gamma_{0}\right)$ are

Table 1 Polynomial coefficient values

\begin{tabular}{crcr}
\hline \hline Coefficient & Value & Coefficient & Value \\
\hline$q_{0}$ & 4.773 & $p_{0}$ & -875.8 \\
$q_{1}$ & -199.6 & $p_{1}$ & 1999.5 \\
$q_{2}$ & 310.5 & $p_{2}$ & -1937.8 \\
$q_{3}$ & -265.8 & $p_{3}$ & 936.8 \\
$q_{4}$ & 120.0 & $p_{4}$ & -226.6 \\
$q_{5}$ & -22.11 & $p_{5}$ & 22.04 \\
\hline \hline
\end{tabular}

Table 2 Thermopile signal computational regions

\begin{tabular}{ccc}
\hline \hline Region & Condition on $\gamma_{0}$ and FOV & $\begin{array}{c}\text { Thermopile } \\
\text { output } \\
\text { function }\end{array}$ \\
\hline 1 & $\frac{\mathrm{FOV}}{2}<\gamma_{0}<\frac{\pi}{2}-\frac{\mathrm{FOV}}{2}$ & $V_{1}\left(\gamma_{0}\right)$ \\
2 & $\frac{\mathrm{FOV}}{2}<\gamma_{0}<\pi-\frac{\mathrm{FOV}}{2}$ and $\frac{\pi}{2}-\frac{\mathrm{FOV}}{2}<\gamma_{0}<\frac{\pi}{2}+\frac{\mathrm{FOV}}{2}$ & $V_{2}\left(\gamma_{0}\right)$ \\
3 & $\frac{\pi}{2}+\frac{\mathrm{FOV}}{2}<\gamma_{0}<\pi-\frac{\mathrm{FOV}}{2}$ & $V_{3}\left(\gamma_{0}\right)$ \\
4 & $\pi-\frac{\mathrm{FOV}}{2}<\gamma_{0}<\frac{\pi}{2}+\frac{\mathrm{FOV}}{2}$ & $V_{4}\left(\gamma_{0}\right)$ \\
5 & $\pi-\frac{\mathrm{FOV}}{2}<\gamma_{0}<\pi+\frac{\mathrm{FOV}}{2}$ and $\frac{\pi}{2}+\frac{\mathrm{FOV}}{2}<\gamma_{0}$ & $V_{5}\left(\gamma_{0}\right)$ \\
6 & $\pi+\frac{\mathrm{FOV}}{2}<\gamma_{0}<2 \pi-\frac{\mathrm{FOV}}{2}$ & $V_{6}\left(\gamma_{0}\right)$ \\
7 & $2 \pi-\frac{\mathrm{FOV}}{2}<\gamma_{0}<2 \pi$ and $\gamma_{0}<\frac{5 \pi}{2}-\frac{\mathrm{FOV}}{2}$ & $V_{7}\left(\gamma_{0}\right)$ \\
8 & $0<\gamma_{0}<\frac{\mathrm{FOV}}{2}$ and $\gamma_{0}>\frac{\pi}{2}-\frac{\mathrm{FOV}}{2}$ & $V_{8}\left(\gamma_{0}\right)$ \\
9 & $0<\gamma_{0}<\frac{\mathrm{FOV}}{2}$ and $\gamma_{0}<\frac{\pi}{2}-\frac{\mathrm{FOV}}{2}$ & $V_{9}\left(\gamma_{0}\right)$ \\
\hline \hline
\end{tabular}

extremely lengthy and cannot be provided here. Also note that FOV is restricted to less than $180^{\circ}$.

A symbolic algebra system was used to solve the integrals shown in the Appendix for closed-form expressions for thermopile voltage output as a function of horizon angle and FOV. The result is nine output functions, each corresponding to specific computational regions. Interestingly, these closed-form expressions consist of polynomial and arcsine terms only.

Projectile orientation is typically modeled using the standard Euler angle sequence composed of yaw angle $\psi$, pitch angle $\theta$, and roll angle $\phi[14]$. Euler pitch and roll angles can be transformed into total horizon angle using the following expression:

$$
\gamma_{0}=\tan ^{-1}\left(\frac{\cos (\theta) \sin (\phi)}{\sqrt{\sin ^{2}(\theta) \sin ^{2}(\phi)+\cos ^{2}(\phi)}}\right)
$$

The preceding model can be used to generate simulated thermopile outputs for a given projectile pitch and roll angle and sensor FOV. Figure $\underline{5}$ shows simulated thermopile outputs for a single roll cycle given a sensor FOV of $120^{\circ}$. The sensor FOV bisector is assumed to be aligned with the $\mathbf{J}_{P}$ axis. Pitch angle is varied to demonstrate how the output signal varies for different projectile orientations, and the scale factor $k_{v}$ in Eq. (3) is assumed to be unity. Note that signal shape is generally sinusoidal as roll angle changes, although at smaller pitch angles the signal becomes compressed at the positive peaks. This is the result of a lower gradient in IR emissivity as the sensor looks straight down. When the projectile is oriented vertically $\left(\theta=90^{\circ}\right)$, the output signal is constant since horizon angle remains constant as roll angle varies. Figure $\underline{6}$ shows how FOV affects thermopile signal response. For various FOVs, thermopile output is shown for one roll cycle assuming zero pitch angle. At small FOVs, the shape of the emissivity curve shown in Fig. 3 is preserved to some extent. At larger FOVs, more IR energy is absorbed by the sensor, tending to increase overall signal amplitude and producing smoother curves. For extremely small FOVs (i.e., a "collimated" FOV), one would expect to obtain a sensor response identical to the stimulus function $y\left(\gamma_{0}\right)$, although this case is not shown here.

The model developed to this point neglects a significant atmospheric phenomenon in the form of atmospheric attenuation and scatter of IR energy as a function of projectile altitude. As altitude increases, more IR energy emitted from the Earth's surface is absorbed and scattered by the atmosphere. Numerous atmospheric scientists have studied this effect and have generated transmission coefficients which characterize total atmospheric attenuation of IR energy as a function of altitude or slant range. Elterman [15] has derived a useful expression for transmission coefficient given by

$$
T(z)=e^{B_{i} z}
$$

where $B_{i}$ changes as a function of wavelength. Figure 7 shows extinction coefficients for various wavelengths for altitudes up to $3000 \mathrm{~m}$. Transmission coefficient can be used to attenuate thermopile output signals as a function of projectile altitude, producing a model of the form given by

$$
V\left(\gamma_{0}\right)=T(z) V_{i}\left(\gamma_{0}\right) \quad \text { where } \gamma_{0} \in \text { region } i
$$

The final model shown in Eq. (7) is a general, stimulus-based model that applies to any thermopile sensor regardless of physical implementation. However, in order to maintain this generality, the model neglects the important effect of sensor temperature. Thermopile sensors, like all thermocouple-based devices, can only measure temperature differentials between hot and cold junctions. Thus, the thermopile sensor temperature also plays a large role in determining output voltage bias. However, methods for sensor temperature compensation are often implementation-specific, and thus the effect is not included here to maintain generality.

\section{B. Flight Simulation Results}

Results are presented to demonstrate simulated thermopile outputs over a full flight trajectory. The example projectile used here is an 


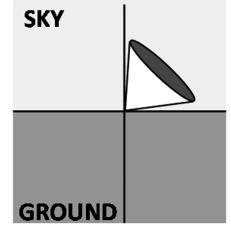

Region 1

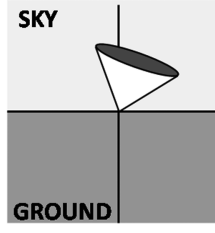

Region 2

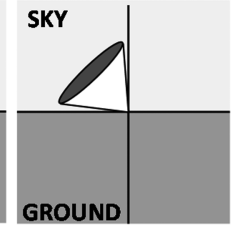

Region 3

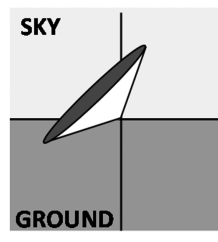

Region 4

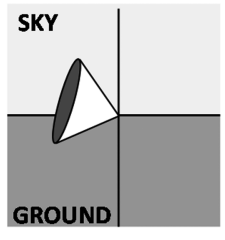

Region 5

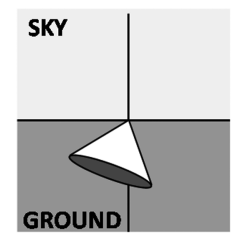

Region 6

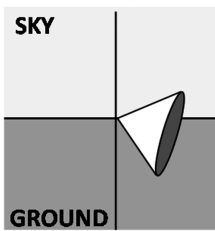

Region 7

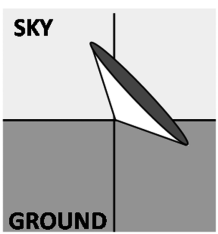

Region 8

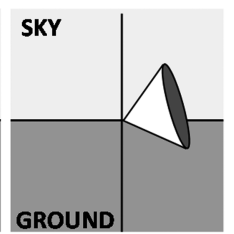

Region 9

Fig. 4 Diagrams of thermopile computational regions. Note that regions 4 and 8 are not valid for FOVs less than $90^{\circ}$.

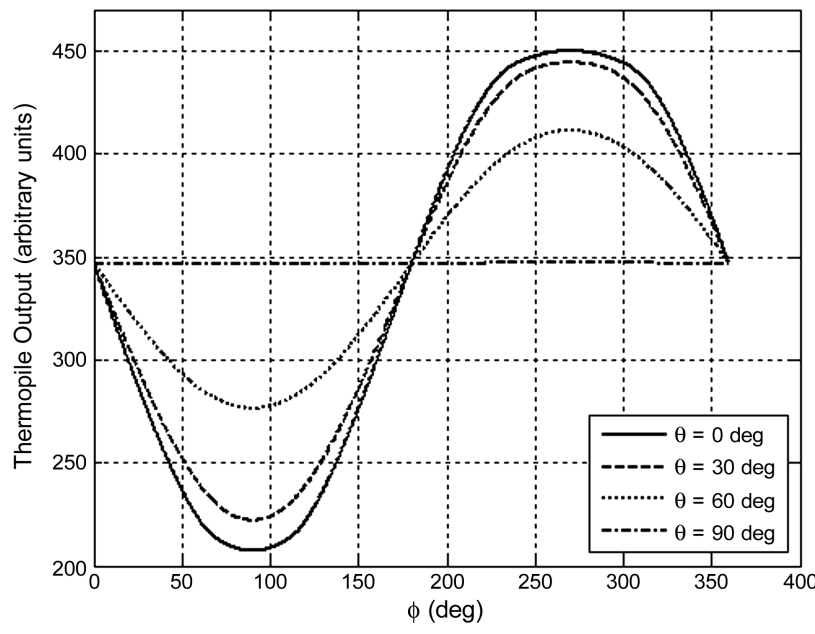

Fig. 5 Thermopile output vs roll angle for various pitch angles.

$81 \mathrm{~mm}$-diam mortar projectile with mass, axial moment of inertia, and transverse moment of inertia given by $5.11 \mathrm{~kg}, 0.0039 \mathrm{~kg}-\mathrm{m}^{2}$, and $0.0757 \mathrm{~kg}-\mathrm{m}^{2}$, respectively. Total projectile length is approximately $0.530 \mathrm{~m}$, and mass center stationline position referenced from the aft end of the round is approximately $37.5 \mathrm{~cm}$. In the example trajectory presented here, the projectile is launched with a quadrant elevation of $800 \mathrm{mrad}$, muzzle velocity of $189.0 \mathrm{~m} / \mathrm{s}$, and zero roll rate. Figure 8 shows altitude-range and deflection-range plots, while Fig. $\underline{9}$ shows pitch angle and roll rate time histories.

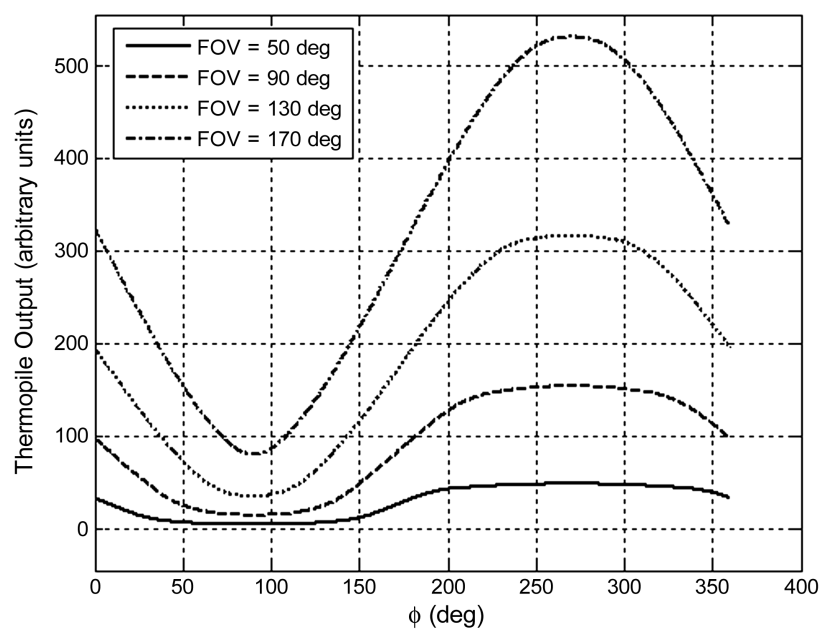

Fig. 6 Thermopile output vs roll angle for various FOVs $(\theta=0)$.

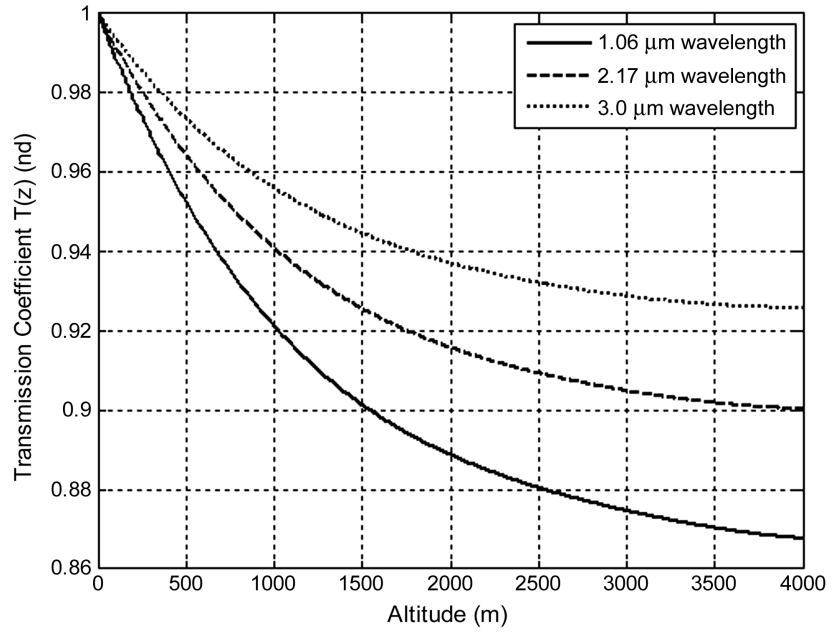

Fig. 7 IR transmission coefficient vs altitude.

Two thermopile sensors are assumed to be fixed on the projectile, both with FOVs of 120 deg. For this example case, sensor noise and time constant are neglected. The FOV bisector of one sensor is aligned with the $\mathbf{J}_{P}$ axis (sensor 1), while the bisector of the second sensor is aligned with $-\mathbf{J}_{P}$ (sensor 2). Both sensor scale factors are assumed to be unity. Figure 10 shows both horizon angle and sensor 1 output time histories, while Fig. 11 shows segments of all time histories. As can be inferred from the single roll cycle results, sensor outputs are approximately sinusoidal in nature with larger
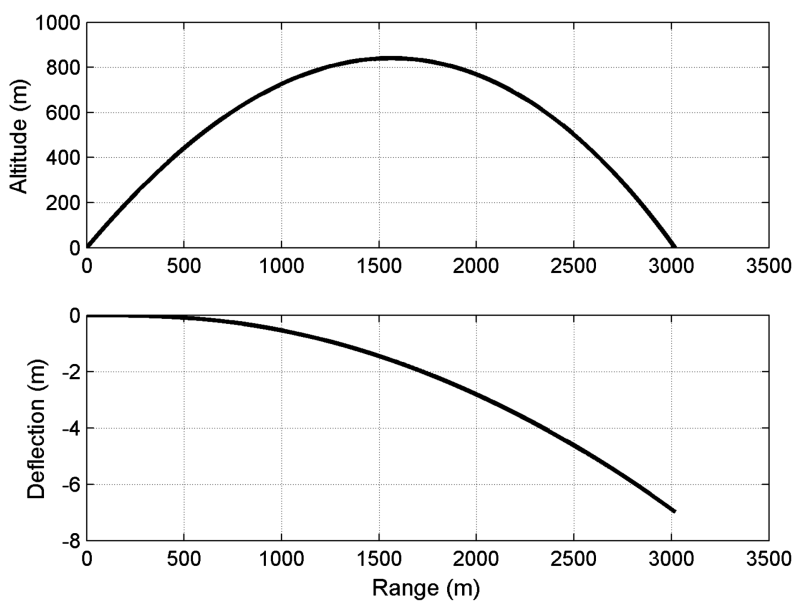

Fig. 8 Altitude and deflection vs range. 

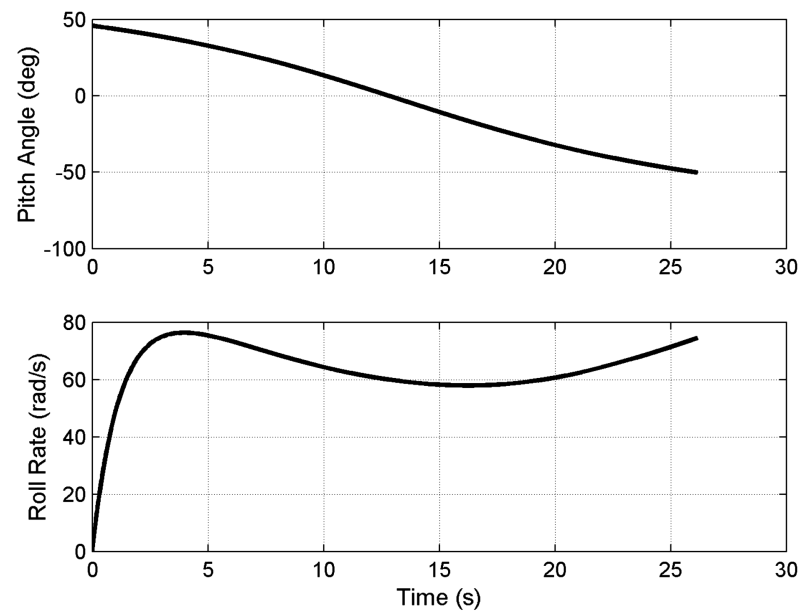

Fig. 9 Pitch angle and roll rate time histories.
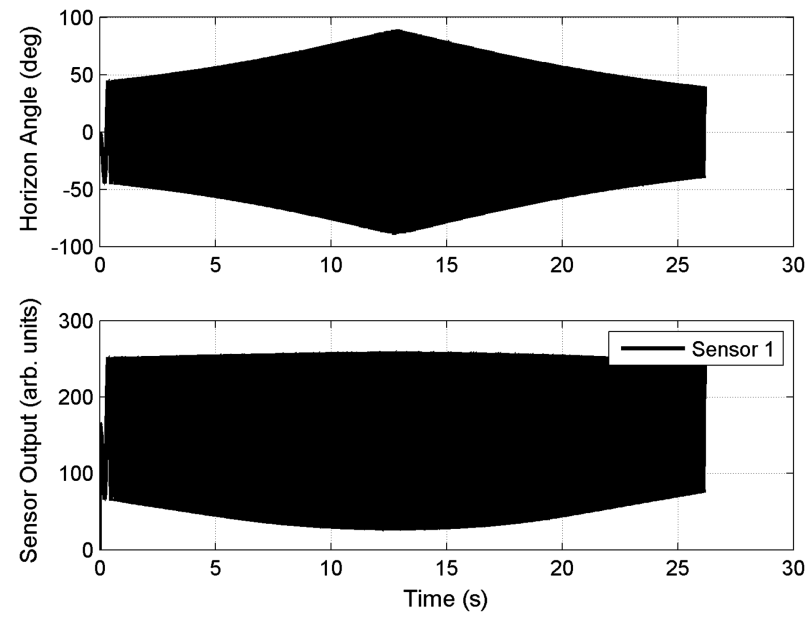

Fig. 10 Horizon angle and thermopile output time histories.

amplitudes occurring near apogee where horizon angle amplitude is largest. Furthermore, outputs from sensors 1 and 2 are 180 deg out of phase with one another due to their orientation on the projectile. Note that for this case the attenuation model corresponding to the $3.0 \mu \mathrm{m}$ trace in Fig. 7 was used. Also note that in Figs. 10 and 11 horizon angle is shown in the interval $-180^{\circ}<\gamma_{0} \leq 180^{\circ}$ rather than $0^{\circ}<$ $\gamma_{0} \leq 360^{\circ}$ solely to create more intelligible plots.
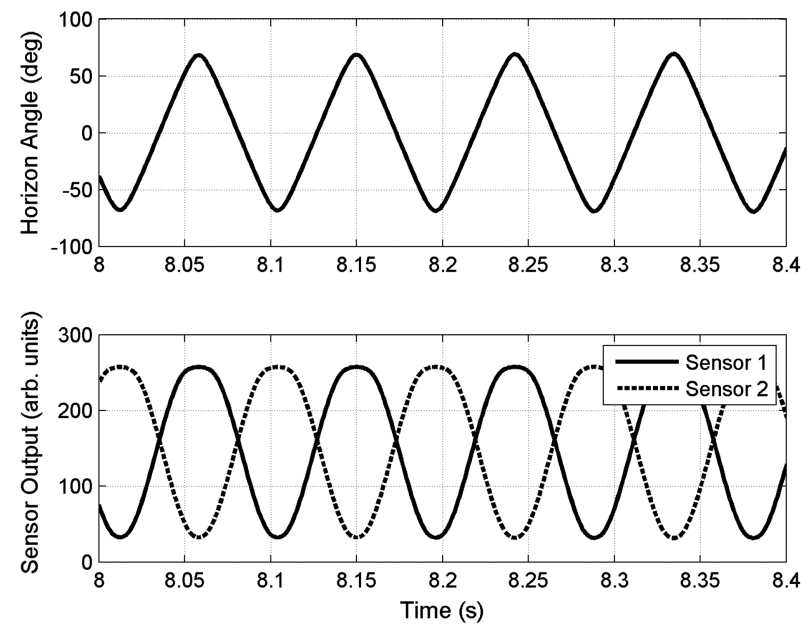

Fig. 11 Segments of horizon angle and thermopile output time histories.
Table 3 Thermopile sensor specifications

\begin{tabular}{lc}
\hline \hline Manufacturer & Heimann sensor GmbH \\
\hline Model & HMS J11F5.5 \\
FOV & $120 \mathrm{deg}$ \\
Operating temp. & -20 to $120 \mathrm{deg} \mathrm{C}$ \\
Time constant & $<6 \mathrm{~ms}$ \\
Noise & $38 \mathrm{nV} / \sqrt{\mathrm{Hz}}$ \\
Sensitivity & $36 \mathrm{~V} / \mathrm{W}$ \\
\hline \hline
\end{tabular}

\section{Experimental Results}

Experimental efforts were conducted to validate thermopile performance in a relevant environment. First, an industry survey was conducted to facilitate selection from a wide variety of available thermopiles. The experimentation was then limited to a single device manufacturer and model. The selected device and salient specifications are shown in Table $\underline{3}$.

This particular model of thermopile requires signal conditioning to amplify output voltage levels to suitable data acquisition voltage levels. Support circuitry was designed, prototyped, and tested for functionality. Next, a miniature circuit board was designed, fabricated, and populated. In this case, it was extremely important to locate high gain low noise amplifier circuitry very close to the sensor. A signal gain of approximately 1000 was implemented with signalto-noise ratio of greater than $90 \mathrm{db}$ for the circuit itself. Noise characterization of the sensor itself was not studied. Additional casing hardware was fabricated to contain both the sensor and support circuitry while providing needed shielding to reduce the possibility of undesired interference. Figure 12 shows a picture of the sensor, signal conditioning board, and mounting hardware. Note that the diameter of the thermopile device (middle component) is approximately $6 \mathrm{~mm}$.

Projectile-borne sensors must resist failure and exhibit operational insensitivity to high- $g$ shock. After assembly, an encapsulating potting compound was used to provide internal rigidity to the components within the casing. Next, an MTS Impac shock simulator was used to subject multiple devices to a variety of orientations and increasing shock levels. Lab-based shock testing of packaged devices demonstrated little or no susceptibility to high- $g$ loading up to and exceeding $12,000 \mathrm{~g}$.

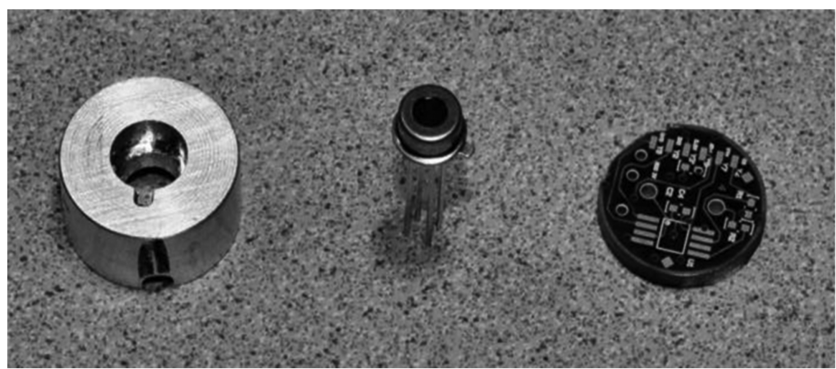

Fig. 12 From left to right: sensor mount, Heimann HMS J11F5.5 thermopile sensor, and signal conditioning circuit board.

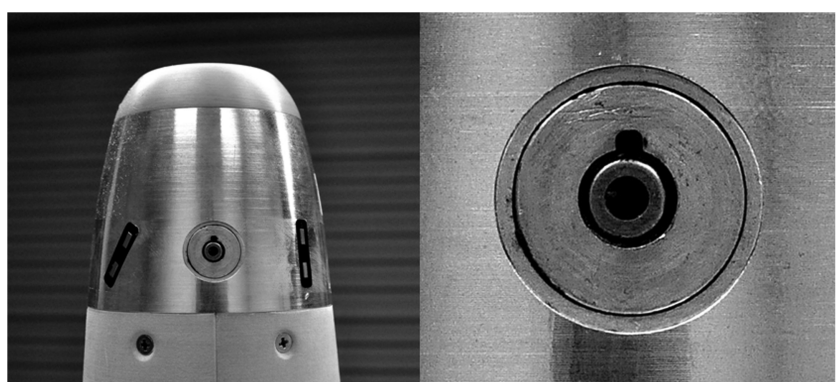

Fig. 13 Thermopile sensor mounted in projectile flight body. 

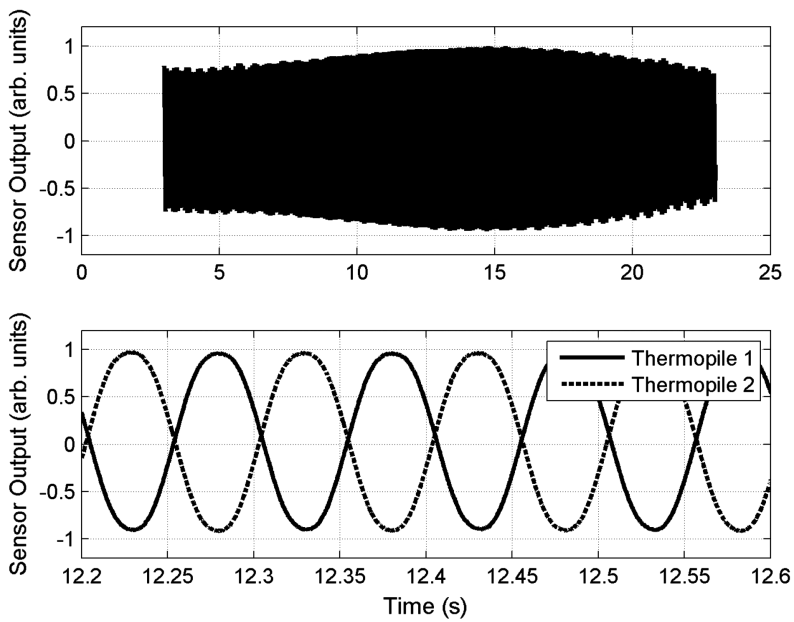

Fig. 14 Normalized, unbiased thermopile data from flight experiment. Time history of outputs from single sensor (top) and segment of data from both sensors (bottom).

Device calibrations were conducted and two devices were installed upon a candidate inert flight body. The two sensors were mounted approximately $180 \mathrm{deg}$ apart from each other around the roll axis. After installation was completed, the thermopile sensor surface was flush with the local surfaces. Figure 13 shows the thermopile sensor mounted on the inert projectile body used for calibration.

Three test projectiles were outfitted with thermopile sensors as shown in Fig. 13 and experimental firings were conducted at Yuma Proving Ground, Arizona, in May 2010. Data from thermopile sensors and independent truth sensors were acquired via an error-free 12-bit S-band telemetry link. Weather during the test was sunny and clear of clouds and terrain was generally flat. Figure 14 shows a time history of the data for the entire flight from one thermopile for one of the test firings, as well as a segment of the time history showing both thermopile outputs. Data from all three tests showed similar behavior. Note that in Fig. 14 data have been normalized and biases have been removed in order to compare signals with those output from the sensor model.

The experimental data shown in Fig. 14 demonstrate several interesting characteristics. First, it is evident from the lower plot that thermopile signals take on the form of a compressed sinusoid with a frequency equal to the projectile roll rate. Second, thermopile signal amplitude is largest near apogee (approximately $16 \mathrm{~s}$ into flight) when the horizon angle undergoes its highest-amplitude oscillation, as seen in the top portion of Fig. 14. Third, outputs from each sensor are $180 \mathrm{deg}$ out of phase with each other due to their mounting configuration. Fourth, signal modulation due to body pitching motion (with frequency of approximately $2 \mathrm{~Hz}$ ) is evident in the upper plot in Fig. 14. Comparing Figs. 10, 11, and 14, it is clear that the model accurately represents the compressed sinusoidal nature of the signal and that it correctly predicts sensor amplitude responses due to pitch angle.

\section{Roll Orientation Estimator}

Both simulation and experimental results demonstrate that thermopile outputs from a rolling projectile provide accurate signals upon which to base orientation estimation. Although sensor outputs are a function of both roll and pitch, it is not feasible to extract pitch information from the signal since variations due to pitch can be easily confused with effects from atmospheric or terrain disturbances. Thus, this section details the development of an extended Kalman filter to determine only roll angle and roll rate in real time using feedback from an arbitrary number of thermopiles. Section IV.A details the estimation algorithm, while Sec. IV.B describes estimation performance using simulated feedback signals. In Sec. IV.C, a trade study is performed to determine how roll orientation accuracy improves as the sensor FOV changes and the number of thermopiles increases.

\section{A. Extended Kalman Filter Design}

An extended Kalman filter is developed to perform real-time roll angle and roll rate estimation. The fundamental assumption underlying the proposed algorithm is that thermopile outputs vary sinusoidally as the projectile rolls, with a frequency corresponding to $\dot{\phi}$ and a time-varying amplitude. Consider a projectile equipped with $n$ thermopiles, each facing radially outward from the spin axis. Neglecting bias components (which can be removed before processing), thermopile outputs from the $i$ th sensor can be approximated as

$$
v_{i}^{*}(t)=A_{i}(t) \sin \left(\phi+\delta_{i}\right) \quad i=1, \ldots, n
$$

where $\delta_{i}$ represents a phase angle determined by sensor orientation with respect to the body. Note that, assuming $\dot{\phi}>0, \delta_{i}=0$ corresponds to a sensor whose FOV bisector is along the $\mathbf{J}_{P}$ axis. Signal amplitude $A_{i}(t)$ depends not only on projectile pitch and roll angle, but also on meteorological conditions, terrain, and sensor scale factor. The unpredictability of these factors means that signal amplitude for each sensor cannot be predicted a priori and must be estimated throughout flight.

The extended Kalman filter, aimed at producing estimates of $\phi$ and $\dot{\phi}$, consists of the following processing steps. First, roll angle at the current time is predicted using the previous estimate according to

$$
\hat{\phi}_{T}^{P}=\hat{\phi}_{T-1}+t_{s} \hat{\dot{\phi}}_{T-1}
$$

where ( ${ }^{\wedge}$ ) denotes the estimated value and $t_{s}$ is estimation time step. The filter error covariance estimate $P$ is also propagated according to

$$
P_{T}^{*}=P_{T-1}+Q
$$

where $Q$ is a scalar gain representing model uncertainty. The predicted roll angle is then used to compute the linearized output matrix $C$ given by

$$
C=\left[\begin{array}{c}
\frac{\partial v_{1}^{*}}{\partial \phi} \\
\frac{\partial v_{2}^{*}}{\partial \phi} \\
\vdots \\
\frac{\partial v_{n}^{*}}{\partial \phi}
\end{array}\right]^{T}=\left[\begin{array}{c}
A_{1} \cos \left(\hat{\phi}_{T}^{P}+\delta_{1}\right) \\
A_{2} \cos \left(\hat{\phi}_{T}^{P}+\delta_{2}\right) \\
\vdots \\
A_{n} \cos \left(\hat{\phi}_{T}^{P}+\delta_{n}\right)
\end{array}\right]^{T}
$$

The output matrix $C$ along with the propagated error covariance $P_{T}^{*}$ is used to compute the Kalman filter gain [16]:

$$
K=P_{T}^{*} C^{T}\left(C P_{T}^{*} C^{T}+R\right)^{-1}
$$

where $R$ is an $n \times n$ diagonal gain matrix representing measurement error covariance. This gain matrix $K$ is then used to correct the previous roll rate estimate according to

$$
\hat{\phi}_{T}=\hat{\phi}_{T}^{P}+K\left\{\begin{array}{c}
v_{1}^{*}-v_{1}^{P} \\
v_{2}^{*}-v_{2}^{P} \\
\vdots \\
v_{n}^{*}-v_{n}^{P}
\end{array}\right\}
$$

where $v_{i}^{*}$ denote actual thermopile measurements at time $T$ and $v_{i}^{P}$ denote predicted thermopile measurements at time $T$. Note that predicted measurements can be obtained using the expression

$$
v_{i}^{P}=\hat{A}_{i} \sin \left(\hat{\phi}_{T}^{P}+\delta_{i}\right)
$$

Finally, the error covariance estimate is updated according to

$$
P_{T}=(1-K C) P_{T}^{*}(1-K C)^{T}+K R K^{T}
$$

The output from Eq. (13), the estimate of the current projectile roll angle, can be used at the next estimator time step as the input to 
Eq. (9). Running estimates of $\dot{\phi}$ can be easily obtained through numerical differentiation techniques. However, if $\dot{\phi}$ estimation is not required, the algorithm will operate well in many sensor configurations using only a rough estimate of $\dot{\phi}$ in Eq. (9) since the measurement update step corrects for prediction inaccuracies.

At each time step, the algorithm must assume a proper amplitude $A_{i}$ for the sine wave signal generated by each thermopile. Signal amplitude can be driven by projectile dynamics, atmospheric conditions, terrain features, and other factors, and thus must be continually updated. This is accomplished by storing the previous $\eta$ measurements for each thermopile and performing peak detection on each time series. These peak values are assumed to be the signal amplitudes for each sensor.

Initialization of the algorithm is accomplished simply by assuming nominal values (which can be extremely inaccurate) for roll angle, roll rate, and signal amplitudes. Initial roll angle error covariance is set at $P=(2 \pi)^{2} \operatorname{rad}^{2}$ to represent large uncertainty as to the initial roll angle. Using proper values for $Q$ and $R$, estimates can be expected to approach reasonable accuracy within one to two roll cycles due to the robust nature of the algorithm.

Kalman filter performance hinges on the ability to perform a suitable measurement update to $\phi$ at each time step. However, note that if the output matrix $C$ shown in Eq. (11) is momentarily zero, all components of the Kalman gain become zero and thus the estimator must rely solely on the prediction step to create an estimate for $\phi$. In this case, any inaccuracies in $\dot{\phi}$ estimation will generate momentary error in roll angle estimates. This condition arises only if all elements of $C$ are zero simultaneously, or equivalently if

$$
\begin{gathered}
\phi+\delta_{1}=(2 m-1) \pi / 2 \\
\phi+\delta_{2}=(2 l-1) \pi / 2 \\
\vdots \\
\phi+\delta_{n}=(2 k-1) \pi / 2
\end{gathered}
$$

This condition occurs if all sensors are achieving their signal maximum or minimum at the same time. The only geometry in which this is practically possible is for the case of two thermopiles mounted facing opposite directions with respect to the projectile plane of symmetry (as shown in Fig. 15). This configuration will experience recurring poor observability twice per roll cycle, since sensor outputs are $180^{\circ}$ out of phase with one another. Note that this configuration happens to be identical to the geometry tested (as described in Sec. III) and thus the experimental data are not suitable for use in algorithm testing and evaluation.

Another condition in which observability suffers is during vertical flight when $\theta=90$ or -90 deg. As shown in Fig. 5, this vertical flight condition results in no variability in thermopile signals as roll angle changes. This scenario is equivalent to all signal amplitudes being zero, at which point the $C$ matrix in Eq. (11) would be zero, resulting in complete unobservability. While theoretically this singularity condition is possible, in practice it is of little consequence for the application discussed here since gun-launched munitions rarely if ever fly a true vertical flight path, even if they are controlled. However, for special scenarios in which flight through $\theta= \pm 90 \mathrm{deg}$ is possible during controlled maneuvers, the processing algorithm discussed here would not provide an accurate estimate for roll angle at or very near this singularity due to low signal amplitudes.

\section{Front View}

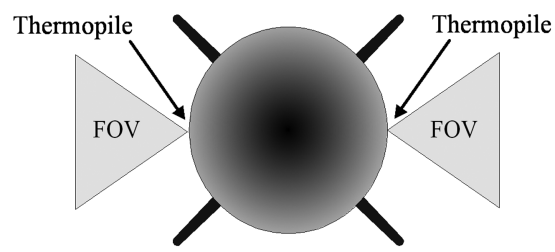

Fig. 15 Front view of projectile equipped with two thermopiles. This sensor orientation will experience recurring poor observability.

\section{B. Example Estimation Results}

Example roll angle and rate estimation results are presented in order to demonstrate algorithm performance given realistic sensor feedback. Simulated thermopile outputs are used for these studies so that algorithm performance can be compared against a known truth source and estimation error can be characterized.

Simulated thermopile outputs were generated for the example projectile and trajectory described in Sec. II. Four thermopiles were assumed to be mounted on the round, evenly spaced about the roll axis with all FOV bisectors perpendicular to the roll axis. Gaussian noise was added to the data to create more realistic sensor outputs. Then, a moving average filter was applied to the data to remove direct current (DC) biases, and the data were normalized between -1 and 1 . Figure 16 shows this conditioned sensor data. Note that thermopile signals are assumed to be available after approximately $3 \mathrm{~s}$ to allow the moving average filter to be applied effectively. The lower plot in Fig. 16 demonstrates that thermopile signals resemble sine waves corrupted by white noise separated by 90 deg phase shifts.

The Kalman filter algorithm was run for this data set using initial guesses of $\phi=1 \mathrm{rad}, \dot{\phi}=100 \mathrm{rad} / \mathrm{s}, P=(2 \pi)^{2} \mathrm{rad}^{2}$, and $A_{i}=$ 0.77 for all four sensors, with estimation results shown in Figs. 1720. Figure 17 shows roll angle estimation error and $3 \sigma$ estimation error covariance bounds output from the Kalman filter. Note that roll angle estimation error is consistently less than about 4 deg in steady state, and that state error covariance bounds generally decrease slightly towards apogee since thermopile signal amplitudes are larger. Figure 18 shows roll rate estimation error obtained from numerical differentiation of roll angle estimates, demonstrating
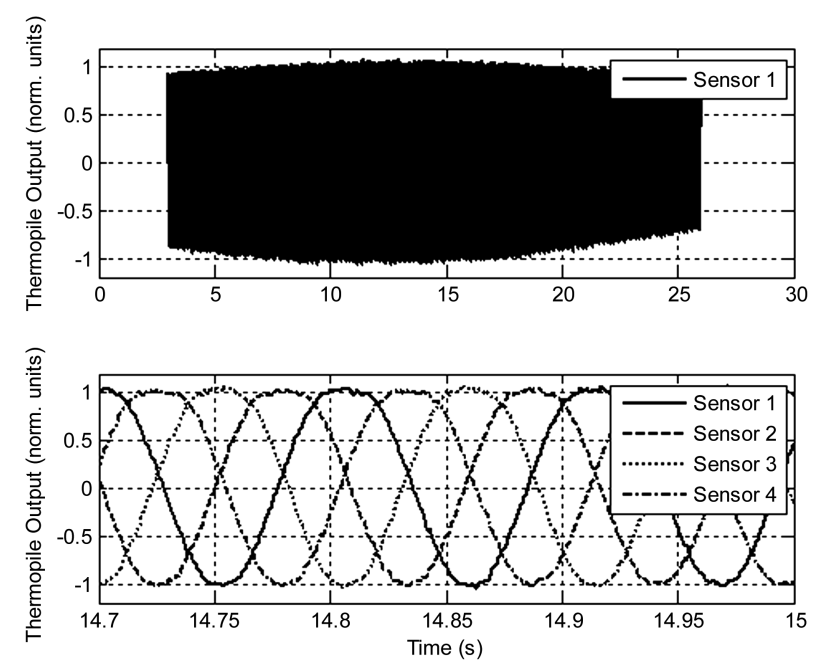

Fig. 16 Thermopile outputs for example estimation case.

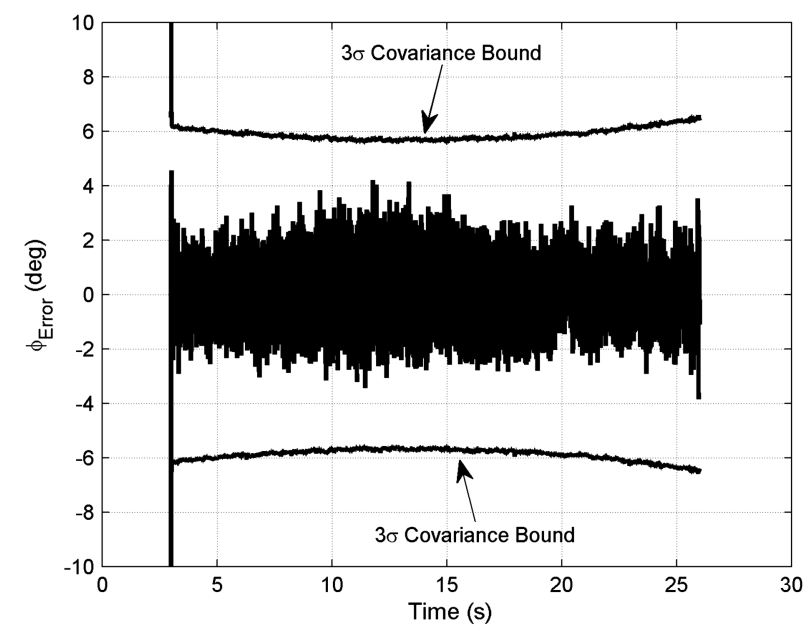

Fig. 17 Roll angle estimation error time history. 


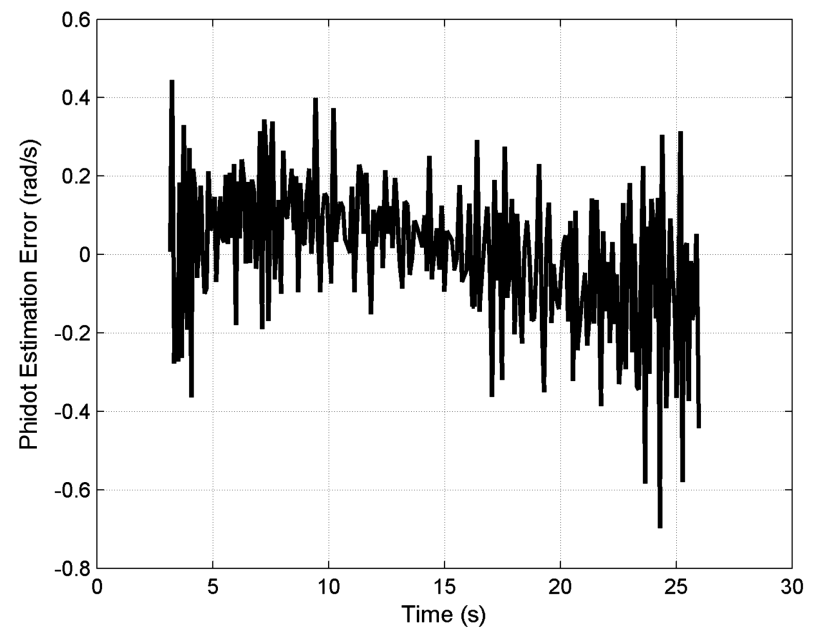

Fig. 18 Roll rate estimation error time history.

accuracy better than $1 \mathrm{rad} / \mathrm{s}$. Figure 19 shows the sine wave amplitude estimate for sensor 1 (aligned with the $-\mathbf{J}_{P}$ axis). Note that this curve is a reasonably accurate approximation to the signal amplitude seen in Fig. 10, with the maximum amplitude occurring at $13 \mathrm{~s}$ (corresponding to apogee). Amplitude estimation curves for the other three sensors are similar and are not shown here. Figure 20 shows roll angle estimation error as a function of actual roll angle,

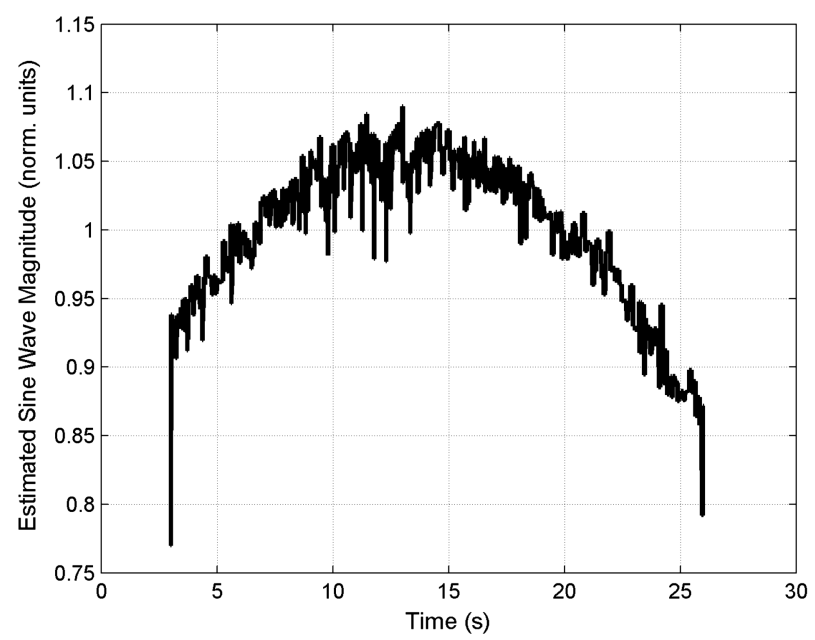

Fig. 19 Sine wave amplitude estimate for sensor 1.

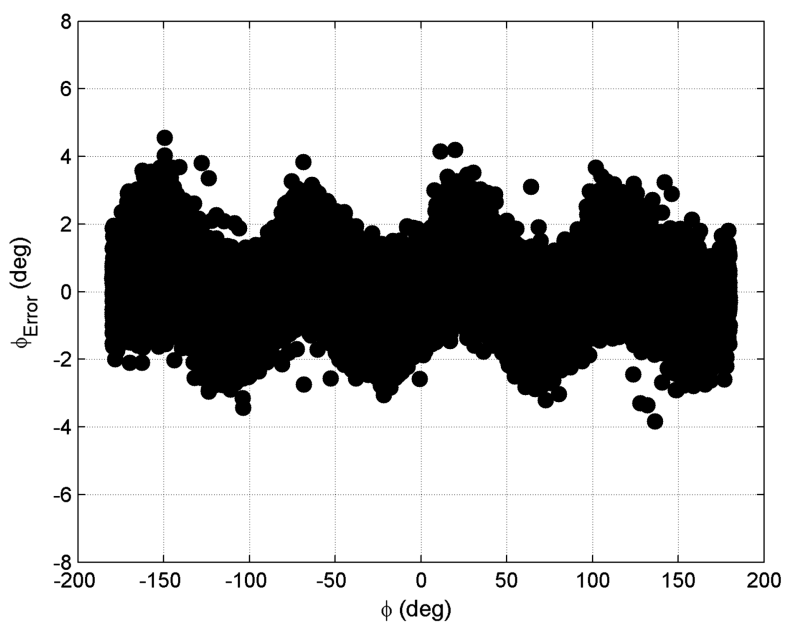

Fig. 20 Roll angle estimation error vs roll angle. and demonstrates that roll estimation error actually occurs in a periodic fashion with respect to roll orientation. Maximum error occurs when the FOV bisectors of each thermopile are farthest from the horizon, and minimum errors are exhibited when the FOV bisectors of two of the sensors are directly aligned with the horizon. This periodic estimation error is the result of the original assumption that the sensor output waveform is sinusoidal. While more detailed models of the waveform can potentially be used to reduce this error, the algorithm simplicity obtained by making this assumption outweigh the cost of larger estimation errors, especially considering that they are bounded to within approximately $4 \mathrm{deg}$.

\section{Field-of-View and Number-of-Thermopiles Trade Study}

A trade study is performed to examine the effect of sensor FOV as well as the number of thermopiles mounted on the body. For sensor FOVs of 95, 125, and $145 \mathrm{deg}$ estimation cases similar to the preceding example were performed. For each sensor FOV, cases were run for varying numbers of thermopiles between 2 and 10. In all cases, thermopiles were assumed to be evenly spaced around the body roll axis (for instance, for the five thermopile cases, each sensor was assumed to be offset by $72 \mathrm{deg}$ around the roll axis from its neighboring sensors). Maximum steady-state and root-mean-square (RMS) roll angle estimation error was calculated for each case. Figures 21 and 22 show the results for RMS and maximum steadystate error, respectively. Note that, as before, sensor noise is incorporated but disturbances due to terrain and atmospheric conditions are beyond the scope of this study and are not considered here.

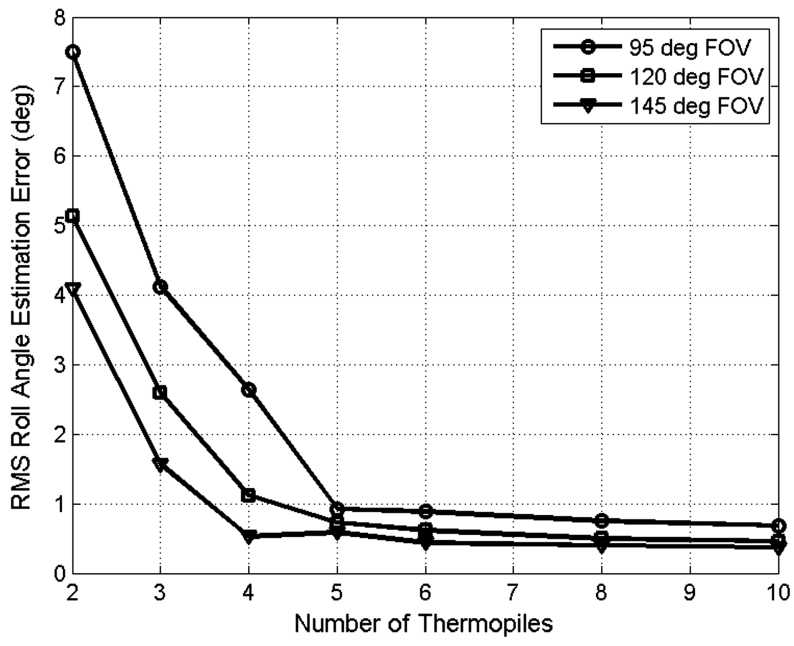

Fig. 21 RMS roll estimation error vs number of thermopiles.

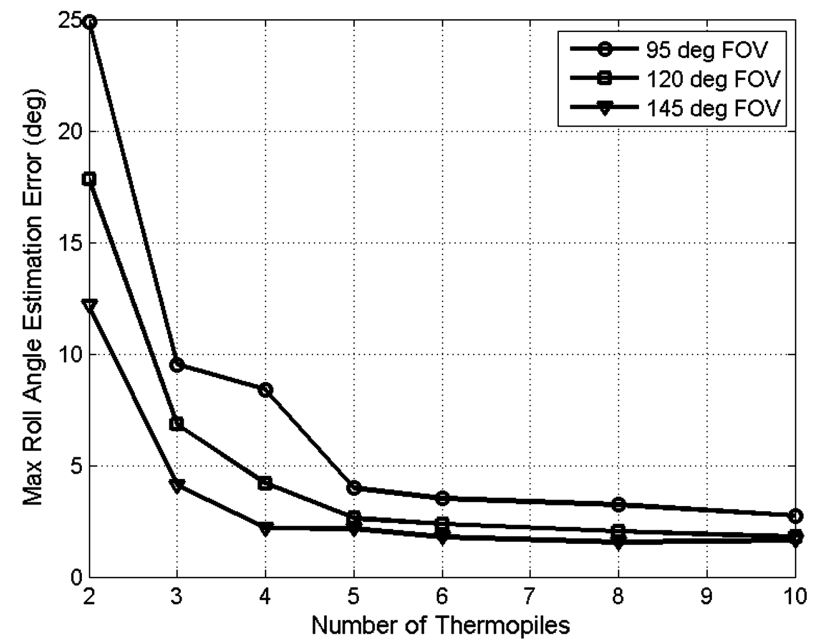

Fig. 22 Maximum steady-state roll estimation error vs number of thermopiles. 
Figures 21 and 22 show that roll angle error decays approximately exponentially as more sensors are incorporated onto the body. Note that in cases in which two sensors were used, maximum and RMS errors were large due to the recurring poor observability as described earlier. Furthermore, both figures demonstrate that there is little benefit to incorporating more than five sensors since errors do not significantly decay beyond this point. Figures 21 and 22 also show

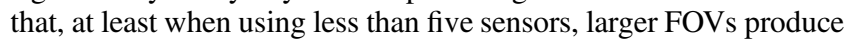
slightly better estimation results. This is because the sinusoidal approximation is better for signals produced by sensors with larger FOVs. Note that for all FOVs, when using more than two sensors roll angle RMS estimation error is consistently below $5 \mathrm{deg}$, at least in this ideal case. These error levels exceed desired performance requirements for most smart projectile GNC applications.

\section{Conclusions}

The feasibility of using wide FOV IR detectors for projectile roll orientation estimation has been examined. A generic thermopile model was created in order to understand sensor response to airframe orientation, altitude, and sensor FOV. Simulated sensor outputs from the model were compared with experimental data from a mortar projectile flight test, showing favorable correlation. An extended Kalman filter algorithm was developed to estimate roll angle and roll rate in real time based strictly on thermopile feedback. Example results show that the algorithm is robust to changes in signal amplitude, and that reasonable accuracy can be expected during clear weather in flat terrain. Trade study results demonstrate that use of three or four body-fixed sensors provides the estimator with sufficient feedback for accurate determination of roll states. Seeing as estimation accuracy is largely driven by how closely thermopile outputs resemble true sinusoids, signal disturbances caused by dense clouds or mountainous terrain can be expected to result in noticeable estimation error. The potential magnitude and the effects of such signal deviations are beyond the scope of this work and will be the subject of future investigations. Overall, thermopile sensors have been shown to be a promising sensor eligible for inclusion in smart projectile orientation sensor packages.

\section{Appendix: Thermopile Output Equations for Each Region}

This Appendix contains integrals used to compute thermopile outputs in all nine regions defined in Sec. II. First, in order to simplify notation, denote

$$
\begin{gathered}
q(\gamma)=q_{5} \gamma^{5}+q_{4} \gamma^{4}+q_{3} \gamma^{3}+q_{2} \gamma^{2}+q_{1} \gamma+q_{0} \\
c(\gamma)=c_{5} \gamma^{5}+c_{4} \gamma^{4}+c_{3} \gamma^{3}+c_{2} \gamma^{2}+c_{1} \gamma+c_{0} \\
p(\gamma)=p_{2} \gamma^{2}+p_{1} \gamma+p_{0}
\end{gathered}
$$

Integrals in each region are presented next:

Region 1:

$$
V_{1}\left(\gamma_{0}\right)=2 k_{v} \int_{\gamma_{0}-\mathrm{FOV} / 2}^{\gamma_{0}+\mathrm{FOV} / 2} q(\gamma) \sqrt{\left(\frac{\mathrm{FOV}}{2}\right)^{2}-\left(\gamma-\gamma_{0}\right)^{2}} \mathrm{~d} \gamma
$$

Region 2:

$$
\begin{gathered}
V_{2}\left(\gamma_{0}\right)=2 k_{v} \int_{\gamma_{0}-\mathrm{FOV} / 2}^{\pi / 2} q(\gamma) \sqrt{\left(\frac{\mathrm{FOV}}{2}\right)^{2}-\left(\gamma-\gamma_{0}\right)^{2}} \mathrm{~d} \gamma \\
+2 k_{v} \int_{\pi / 2}^{\gamma_{0}+\mathrm{FOV} / 2} c(\gamma) \sqrt{\left(\frac{\mathrm{FOV}}{2}\right)^{2}-\left(\gamma-\gamma_{0}\right)^{2}} \mathrm{~d} \gamma
\end{gathered}
$$

Region 3:

$$
V_{3}\left(\gamma_{0}\right)=2 k_{v} \int_{\gamma_{0}-\mathrm{FOV} / 2}^{\gamma_{0}+\mathrm{FOV} / 2} c(\gamma) \sqrt{\left(\frac{\mathrm{FOV}}{2}\right)^{2}-\left(\gamma-\gamma_{0}\right)^{2}} \mathrm{~d} \gamma
$$

Region 4:

$$
\begin{aligned}
& V_{4}\left(\gamma_{0}\right)=2 k_{v} \int_{\gamma_{0}-\mathrm{FOV} / 2}^{\pi / 2} q(\gamma) \sqrt{\left(\frac{\mathrm{FOV}}{2}\right)^{2}-\left(\gamma-\gamma_{0}\right)^{2}} \mathrm{~d} \gamma \\
& +2 k_{v} \int_{\pi / 2}^{\pi} c(\gamma) \sqrt{\left(\frac{\mathrm{FOV}}{2}\right)^{2}-\left(\gamma-\gamma_{0}\right)^{2}} \mathrm{~d} \gamma \\
& 2 k_{v} \int_{\pi}^{\gamma_{0}+\mathrm{FOV} / 2} p(\gamma) \sqrt{\left(\frac{\mathrm{FOV}}{2}\right)^{2}-\left(\gamma-\gamma_{0}\right)^{2}} \mathrm{~d} \gamma
\end{aligned}
$$

Region 5:

$$
\begin{gathered}
V_{5}\left(\gamma_{0}\right)=2 k_{v} \int_{\gamma_{0}-\mathrm{FOV} / 2}^{\pi} c(\gamma) \sqrt{\left(\frac{\mathrm{FOV}}{2}\right)^{2}-\left(\gamma-\gamma_{0}\right)^{2}} \mathrm{~d} \gamma \\
+2 k_{v} \int_{\pi}^{\gamma_{0}+\mathrm{FOV} / 2} p(\gamma) \sqrt{\left(\frac{\mathrm{FOV}}{2}\right)^{2}-\left(\gamma-\gamma_{0}\right)^{2}} \mathrm{~d} \gamma
\end{gathered}
$$

Region 6:

$$
V_{6}\left(\gamma_{0}\right)=2 k_{v} \int_{\gamma_{0}-\mathrm{FOV} / 2}^{\gamma_{0}+\mathrm{FOV} / 2} p(\gamma) \sqrt{\left(\frac{\mathrm{FOV}}{2}\right)^{2}-\left(\gamma-\gamma_{0}\right)^{2}} \mathrm{~d} \gamma
$$

Region 7:

$$
\begin{aligned}
& V_{7}\left(\gamma_{0}\right)=2 k_{v} \int_{\gamma_{0}-\mathrm{FOV} / 2}^{2 \pi} p(\gamma) \sqrt{\left(\frac{\mathrm{FOV}}{2}\right)^{2}-\left(\gamma-\gamma_{0}\right)^{2}} \mathrm{~d} \gamma \\
& +2 k_{v} \int_{0}^{\gamma_{0}+\mathrm{FOV} / 2-2 \pi} q(\gamma) \sqrt{\left(\frac{\mathrm{FOV}}{2}\right)^{2}-\left(\gamma-\gamma_{0}+2 \pi\right)^{2}} \mathrm{~d} \gamma
\end{aligned}
$$

Region 8:

$$
\begin{aligned}
& V_{8}\left(\gamma_{0}\right)=2 k_{v} \int_{\gamma_{0}-\mathrm{FOV} / 2+2 \pi}^{2 \pi} p(\gamma) \sqrt{\left(\frac{\mathrm{FOV}}{2}\right)^{2}-\left(\gamma-\gamma_{0}-2 \pi\right)^{2}} \mathrm{~d} \gamma \\
& +2 k_{v} \int_{0}^{\pi / 2} q(\gamma) \sqrt{\left(\frac{\mathrm{FOV}}{2}\right)^{2}-\left(\gamma-\gamma_{0}\right)^{2}} \mathrm{~d} \gamma \\
& +2 k_{v} \int_{\pi / 2}^{\gamma_{0}+\mathrm{FOV} / 2} c(\gamma) \sqrt{\left(\frac{\mathrm{FOV}}{2}\right)^{2}-\left(\gamma-\gamma_{0}\right)^{2}} \mathrm{~d} \gamma
\end{aligned}
$$

Region 9:

$$
\begin{aligned}
& V_{9}\left(\gamma_{0}\right)=2 k_{v} \int_{\gamma_{0}-\mathrm{FOV} / 2+2 \pi}^{2 \pi} p(\gamma) \sqrt{\left(\frac{\mathrm{FOV}}{2}\right)^{2}-\left(\gamma-\gamma_{0}-2 \pi\right)^{2}} \mathrm{~d} \gamma \\
& +2 k_{v} \int_{0}^{\gamma_{0}+\mathrm{FOV} / 2} q(\gamma) \sqrt{\left(\frac{\mathrm{FOV}}{2}\right)^{2}-\left(\gamma-\gamma_{0}\right)^{2}} \mathrm{~d} \gamma
\end{aligned}
$$

\section{References}

[1] "Spacecraft Earth Horizon Sensors," NASA Rept. NASA SP-8033, Dec. 1969.

[2] Astheimer, R. W., "High Precision Attitude Determination by Sensing the Earth and Lunar Horizon in the Infrared," Automatica, Vol. 7, No. 1, 1971, pp. 83-88. doi:10.1016/0005-1098(71)90082-3

[3] Doctor, A. P., "Earth Sensor for Satellite with Radiance Compensation," U.S. Patent No. 5,477,052, filed Dec. 1995. 
[4] Grassi, M., "Attitude Determination and Control for a Small Remote Sensing Satellite," Acta Astronautica, Vol. 40, No. 9, 1997, pp. 675681.

doi:10.1016/S0094-5765(97)00023-4

[5] Smith, E. Q., Jr., Marson, M., Hewitt, W., and Hage, W., "Infrared Horizon Sensor for Missile Attitude Control," U.S. Patent No. 3,311,747, filed Dec. 1963

[6] Baxter, W. F., "Simulation of an Attitude Determining System Utilizing Magnetometers and Earth's Horizon Sensor," U.S. Army Missile Command Rept. No. RG-TR-65-11, Redstone Arsenal, AL, May 1965.

[7] Gwozdecki, J. A., "Aircraft Attitude Sensor and Feedback Control System," U.S. Patent No. 6,181,989, filed Jan. 2001.

[8] Taylor, B., Bil, C., Watkins, S., and Egan, G., "Horizon Sensing Attitude Stabilisation: A VMC Autopilot," 18th International UAV Systems Conference, Bristol, England, U.K., 2003.

[9] Cornall, T. D., Egan, G., and Price, A., "Aircraft Attitude Estimation from Horizon Video," Electronics Letters, Vol. 42, No. 13, June 2006, pp. 744-745.

[10] Herrmann, P., and Bil, C., "Simulation and Flight Test of a Temperature Sensing and Stabilisation System," The Aeronautical Journal, Vol. 109,
April 2005, pp. 167-176.

[11] Chahl, J., Thakoor, S., Le Bouffant, N., Stange, G., Srinivasan, M., Hine, B., and Zornetzer, S., "Bioinspired Engineering of Exploration Systems: A Horizon Sensor/Attitude Reference System Based on the Dragonfly Ocelli for Mars Exploration Applications," Journal of Robotic Systems, Vol. 20, No. 1, 2003, pp. 35-42. doi:10.1002/rob.10068

[12] Fraden, J., Handbook of Modern Sensors: Physics, Design, and Applications, 3rd ed., Springer-Verlag, New York, 2004, Chap. 14.7, pp. 481-497.

[13] Egan, G. K., and Taylor, B., "Characterisation of Infrared Sensors for Absolute Unmanned Aerial Vehicle Attitude Determination," Monash Univ. TR MECSE-2007, Melbourne, Australia, 2007.

[14] Etkin, B., and Reid, L. D., Dynamics of Flight: Stability and Control, Wiley, New York, 1996, p. 98.

[15] Elterman, L., "Atmospheric Attenuation Model, 1964, in the Ultraviolet, Visible, and Infrared Regions for Altitudes up to $50 \mathrm{~km}$," Air Force Cambridge Research Labs. Rept. No. AFCRL-64-740, Hanscom Field, MA, Sept. 1964.

[16] Zarchan, P., and Musoff, H., Fundamentals of Kalman Filtering: A Practical Approach, AIAA, Reston, VA, 2005, p. 258. 